\title{
Using Generalized Weighted Minimum Entropy Deconvolution Algorithm to Improve The Resolution of Ultrasonic Guided Wave Testing
}

\author{
Haichao Cai ${ }^{1,2}$, Chunguang $\mathrm{Xu}^{1}$, Qinxue Pan $^{1 *}$ and Hongjuan $\mathrm{Yan}^{1}$ \\ ${ }^{1}$ Key Laboratory of Fundamental Science for Advanced Machining, Beijing \\ Institute of Technology, Beijing 100081, China \\ ${ }^{2}$ School of Mechatronics Engineering, Henan University of Science and \\ Technology, Luoyang 471000, China \\ chc1226@haust.edu.cn,xucg@bit.edu.cn,panqx@bit.edu.cn
}

\begin{abstract}
Ultrasonic guided wave detection technology has numerous advantages compared with body waves. Accurately obtaining the time-of-arrival (TOA) or time-of-flight (TOF) of the ultrasonic guided wave signal is critical for the detection and location of defect because of the frequent use of the low frequency guided wave probe in testing. Thus, guaranteeing the propagation over a long distance and increasing the resolution of defect detection become the key points. This study adds the weighted nonlinear transformation functions to the minimum entropy deconvolution (MED). By adjusting the corresponding parameters, it can enhance a weak signal of small defects and suppress the noise signal. The experimental results indicate that this method can accurately obtain TOA to enhance the resolution of defect detection and can improve the speed of convergence effectively compared with MED.
\end{abstract}

Keywords: Ultrasonic Guided Wave; Deconvolution; Resolution; Weighted Nonlinear Transformation

\section{Introduction}

In the past few decades, ultrasonic guided wave testing has made significant progress, which is widely applied in the non-destructive test and evaluation of tubes, rods, pressure vessels and other structures [1-3]. Ultrasonic guided wave is a kind of ultrasonic wave that is restricted by its structure boundary in the transmission of bounded structural, for the reason, it is called guided wave. Although both the body wave and the guided wave are controlled by the same set of partial differential equations, the latter is controlled by the additional boundary conditions. An ultrasonic guided wave has the advantage of long distance propagation and detected complex structure. In the ultrasonic guide wave propagation process, an echo signal, which contains numerous information, will change because of the discontinuity or property changes of the structure. The characteristics of echoes are determined by the physical characteristics of the reflector, such as location, size, and direction [4-6]. We can study the echo signal characteristics by constructing the ultrasonic echo signal model. The ultrasonic guide wave signal can be seen as the convolution of a system time response and ultrasonic reflection sequence. Obtaining the time-of-arrival (TOA) or time-of-flight (TOF) is important to pinpoint the location of defects accurately. The ultrasonic guide wave detection hope to be able to propagate in a long distance and precisely detect the small defects. The ultrasonic guide wave detection resolution is mainly determined by the transducer frequency and its design. Although a

Corresponding Author 
higher frequency of the transducer can obtain better detection resolution, the rapid attenuation of the ultrasonic signal in the propagation process is at the cost of sacrificing the propagation distance. Ultrasonic guide wave detection currently selects the low frequency transducers, which leads to a low defect detection resolution. In addition, frequency dispersion in the transmission process causes considerable difficulties to the TOA or TOF extraction [7-8].

The methods of obtaining the TOF estimation include cross-correlation, threshold comparison, and sliding window, and so on. However, the cross-correlation method requires the signal noise to be a Gauss white noise. Furthermore, compared with the reference waveform, the frequency domain of the received signal has no change, and the dispersion of the guided wave signal constrains the accuracy of the cross-correlation method to solve time arrival [9-10]. In the detection process, the collected ultrasonic guided wave signal can be expressed by the convolution model of the transducer impulse response and reflection sequence [11]. Therefore, the deconvolution method can effectively restore the reflection sequence, improve the defect detection resolution, and obtain an accurate TOA or TOF expression.

The traditional deconvolution method for minimum phase or linear systems can play a good role in the reflection sequence recovery. When the system is nonlinear or has a non-minimum phase, deconvolution will appear as an abnormal phenomenon [12-13]. However, the ultrasonic guided wave detection signal has two characteristics, namely, non-minimum phase and nonlinear. The minimum entropy deconvolution (MED) proposed by Wiggins [14] is a method that does not require making any assumptions regarding the system phase. If the output sequence is a sparse spike pulse, then such output can be a good solution to the problem of non-minimum phase and nonlinear system deconvolution. However, the MED output sequence is not an actual sparse spike pulse sequence because it seriously affects the reflection sequence resolution. Furthermore, the nature of MED is to determine the inverse filter of the smallest entropy of the output sequence through iteration. The iteration process makes the speed of convergence and computation times of the algorithm become another problem to consider. To enhance the deconvolution output sequence sparsity and to improve the convergence speed, a modified MED algorithm has been applied in the ultrasonic nondestructive testing [11-15]. These methods have a good effect on the improvement of the deconvolution output sequence sparsity and the acceleration of the convergence speed.

This study presents a nonlinear transformation function to construct a generalized weighted MED (GWMED) algorithm, which is applied in the ultrasonic guided wave defect detection. In the process of deconvolution, through adjusting the parameters of nonlinear transform, the algorithm can achieve the deconvolution of guided wave signal. Compared with other deconvolution algorithm, the output sequence of GWMED algorithm becomes sparser and the convergence speed of algorithm becomes faster. The algorithm plays a good role in overcoming the influence of the guided wave dispersion effect and the precise extraction of TOA.

This paper is organized as follows: Section 2 describes the ultrasonic guided wave signal convolution model. Section 3 introduces the minimum entropy deconvolution principle. In Section 4, we modified the minimum entropy deconvolution algorithm. Section 5 is the experimental results and discussion. A conclusion is given at the end.

\section{Ultrasonic Guided Wave Signal Convolution Model}

The guided wave signal involves a convolution process among high-voltage pulses and transducers, the measured object (pipes), defects, test systems, and the other aspects of the integrated transfer function [16], assuming the echo signal received by the ultrasonic transducer is expressed as:

$$
x(t)=S_{d}(t) * H(t) * H_{i n v}(t) * d(t) * S_{m}(t)+\sigma(t)
$$


In Eq. (1), $S_{d}(t)$ is the electrical-mechanical response of the guide wave transducer. $H(t)$ is the forward direction transmission characteristics from the guided wave transducer to the defects, which has connections with the wave guide structure, size, shape, location, and the number of defects. $H_{i n v}(t)$ is the reverse transmission characteristics from the defects to the guided wave transducers, which has connections with the amplitude, phase, and propagation distance of the waveform. $d(t)$ is the reflection response of the defects, which is the reflection sequence. $S_{m}(t)$ is the mechanical-electrical response of the guided wave transducer. $\sigma(t)$ is the noise.

In Eq. (1), $S_{d}(t), H(t), H_{i n v}(t)$ and $S_{m}(t)$ are the response characteristics of every section on the guided wave detection system, which can be expressed as the following equation:

$$
h(t)=S_{d}(t) * H(t) * H_{i n v}(t) * S_{m}(t)
$$

Substitute Eq. (2) into Eq. (1), we can obtain the convolution model of the guided wave signals as:

$$
x(t)=h(t) * d(t)+\sigma(t)
$$

From the convolution model of the guided wave, if we eliminate the influence of noise $\sigma(t)$ and system response $h(t)$ from the collected signals $x(t)$, then we can recover the reflecting sequences $d(t)$ to improve the guided wave inspection resolution; thus, the dispersion effect on the TOA extraction is reduced.

\section{Minimum Entropy Deconvolution Principle}

From the definition of entropy in information theory, the better the sparsity of the guided wave signal sequence, the entropy is smaller. The discretization of the guided waves signal convolution models will obtain [11-17]:

$$
x(n)=h(n) * d(n)+\sigma(n)
$$

If we do not consider the influence of noise after convoluting, then the entropy of $d(n)$ as the sparse reflection sequence and system response $h(n)$ will further increase. An inverse filter $f(n)$ can recover $d(n)$ from the sequence $x(n)$. Thus, the better the performance of the filter $f(n)$, the simpler the reflection sequence $d(n)$ will be. In addition, the entropy will be smaller. MED determines the optimal inverse filter and makes the entropy change for the smallest. Wiggins utilizes the norm of sequence $x(n)$ as the method to evaluate the sequence entropy size. Furthermore, he designates the $O_{2}^{4}(f(n))$ as the objective function to obtain the optimal results. MED is the maximum of the inverse filter norm $O_{2}^{4}(f(n))$, that is,

$$
\partial O_{2}^{4}(f(n)) / \partial f(n)=0
$$

If the length of the inverse filter is $L$, then $d(n)=f(n) * x(n)$ can be expressed as:

$$
d(n)=\sum_{m=1}^{L} f(m) x(n-m)
$$

Eq. (5) can obtain: 


$$
\frac{\sum_{n=1}^{N} d^{2}(n)}{\sum_{n=1}^{N} d^{4}(n)} \sum_{n=1}^{N} d^{3}(n) x(n-m)=\sum_{q=1}^{L} f(q) \sum_{n=1}^{N} x(n-l) x(n-p)
$$

Eq. (7) can be expressed in the following matrix form:

$$
\boldsymbol{b}=\boldsymbol{A f}
$$

In Eq. (8), $\boldsymbol{b}=\left[\frac{\sum_{n=1}^{N} d^{2}(n)}{\sum_{n=1}^{N} d^{4}(n)} \sum_{n=1}^{N} d^{3}(n) x(n-m)\right]^{T}, \quad \boldsymbol{A}=\sum_{n=1}^{N} x(n-l) x(n-p)$ is the autocorrelation matrix of $x(n)$. The inverse filter matrix can be obtained through the iterative computation of Eq. (8):

$$
\boldsymbol{f}=\boldsymbol{A}^{-1} \boldsymbol{b}
$$

\section{Generalized Weighted Minimum Entropy Deconvolution}

The purpose of MED is to determine an optimal inverse filter coefficient through iteration and to highlight the few large spikes in the sequence, which makes the sparseness of the reflection sequence the best. However, the algorithm is vulnerable to noise; thus, the sparsity of the iterative output results is unexpected. The algorithm reduces the inspection resolution particularly for the weak signal of the small defects.

This study proposes an adjustable parameter nonlinear transformation to obtain the sparsest solutions. We can increase the sparsity of the iterative output sequence and reduce the effect of the noises through nonlinear transformation. The adjustable parameters of the nonlinear transfer functions are as follows:

$$
\hat{d}(n)=\frac{w(n)}{\sum_{i=1, i \neq n}^{N} w(i)}-\frac{w(n)}{\sum_{i=1, i \neq n}^{N} w(i)}
$$

In Eq. $(10), w(n)=a(d(n))^{b}, a, b$ are the adjusting factors. The value of regulatory factor is larger than 1 . If the strong component amplitude of the signal is larger than 1 , then adjusting the value of $b$ can make the strength component comparison larger. If the strong component amplitude of the signal is less than 1 , then further adjusting the value of $a$ can achieve the same effect. After the transformation of $w(n)$, regulators $a, b$ can make the strong component larger and the weak component smaller of the time series $d(n)$, which increases the contrast between sharp pulse and noise.

If $d(n)$ is the value generated by the noise, compared with the larger sharp pulse, then its value is relatively small. Thus,

$$
\frac{w(n)}{\sum_{i=1, i \neq n}^{N} w(i)}-\frac{w(n)}{\sum_{i=1}^{N} w(i)} \approx 0
$$

If $d(n)$ is the value generated by the few large sharp pulses, then 
$\frac{w(n)}{\sum_{i=1, i \neq n}^{N} w(i)}-\frac{w(n)}{\sum_{i=1}^{N} w(i)}>0$

If the difference between the few large sharp pulses and noise is larger, then adjustments in $a, b$ can make such difference even larger. If the few large sharp pulses and noise do not have a slight difference between them, then adjusting $a, b$ can increase the difference. They can effectively highlight the sharp pulse of time sequence, which achieves the purpose of noise suppression.

Considering the influence of noise, the iterative computation steps of the GWMED algorithm are as follows:

1. Estimate the variance $\varsigma$ of signal;

2. Calculate $x(n)$ 's correlation matrix $\boldsymbol{A}$ and its inverse matrix, in which the coefficient of the initial filter $f^{0}$ is 1 ;

3. Compute the output signal $d(n)=f^{0} * x(n)$;

4. Calculate $w(n)$ and apply to the transformation $d(n)=d(n)$;

5. Calculate matrix $\boldsymbol{b}$;

6. Update the filter coefficients $f^{1}=\left(\boldsymbol{A}-\varsigma^{2} \boldsymbol{I}\right)^{-1} \boldsymbol{b}$

7. Calculate the iterative criterion, and make the kurtosis value of signal as the criterion.

The calculation of the kurtosis is expressed as $k(j)=\sum_{1}^{n} d_{n}^{4} /\left(\sum_{1}^{n} d_{n}^{2}\right)^{2}(j$ is the iterations number). Assess whether $k(j)-k(j-1)$ is smaller than the given threshold. If it is smaller than the given threshold, then the iteration should stop. Otherwise, the iteration should continue and return to step 3.

\section{Experimental Results and Discussion}

The experimental object is a gas pipeline, which is $1000 \mathrm{~mm}$ long, has a $273 \mathrm{~mm}$ outside diameter, and $8 \mathrm{~mm}$ wall thickness. The pipe material is X70 steel. The outer surface of the pipe prefabricates the artificial defect, with a rectangular shape, and is made with cutter. The defect is $20 \mathrm{~mm}$ long, $2 \mathrm{~mm}$ wide, and $3 \mathrm{~mm}$ deep, and is $128 \mathrm{~mm}$ from the transducer in the axial direction. The experimental testing system is composed of a center frequency of $0.5 \mathrm{MHz}$ wedge ultrasonic probes, AD-IPR1210 pulsed ultrasonic transceiver card, four-channel 4104B-Tektronix digital storage oscilloscope, and industrial personal computer (IPC). Figure 1, shows the schematic diagram of the experimental system. The experiment uses the oblique wedge technology to inspire $L(0,2)$ guided wave mode for testing. Data are recorded in the form of A-SCAN. Figure 2 (a), shows the original signal waveforms.
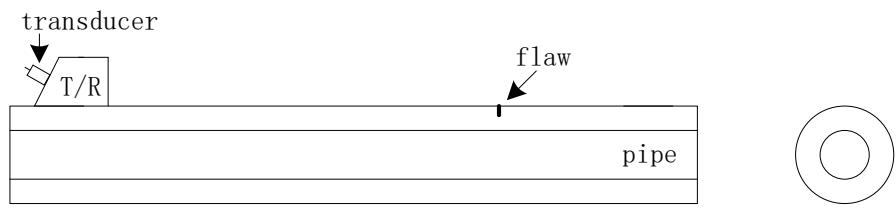

Figure 1. Schematic Diagram of Experiment One 


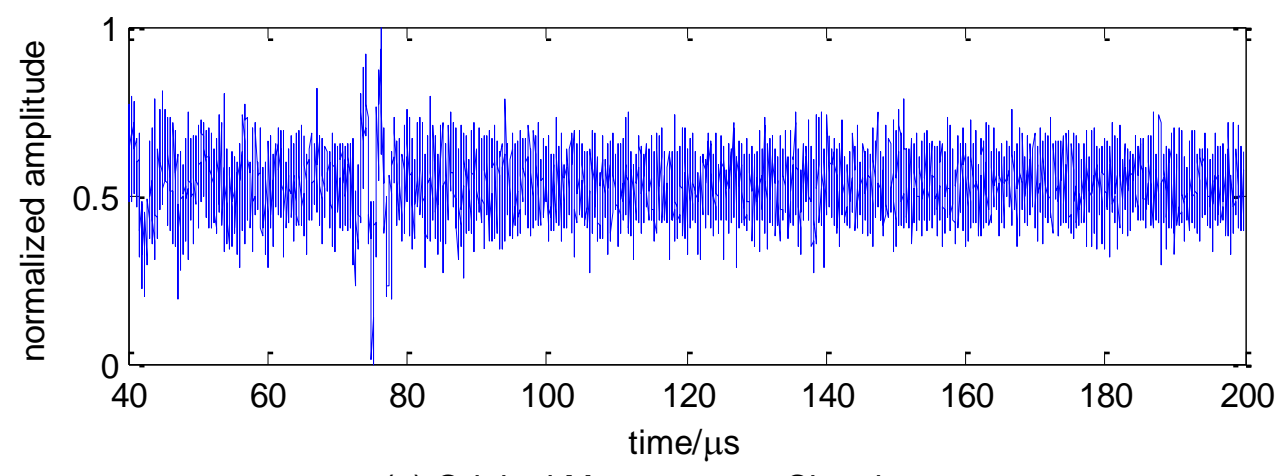

(a) Original Measurement Signal

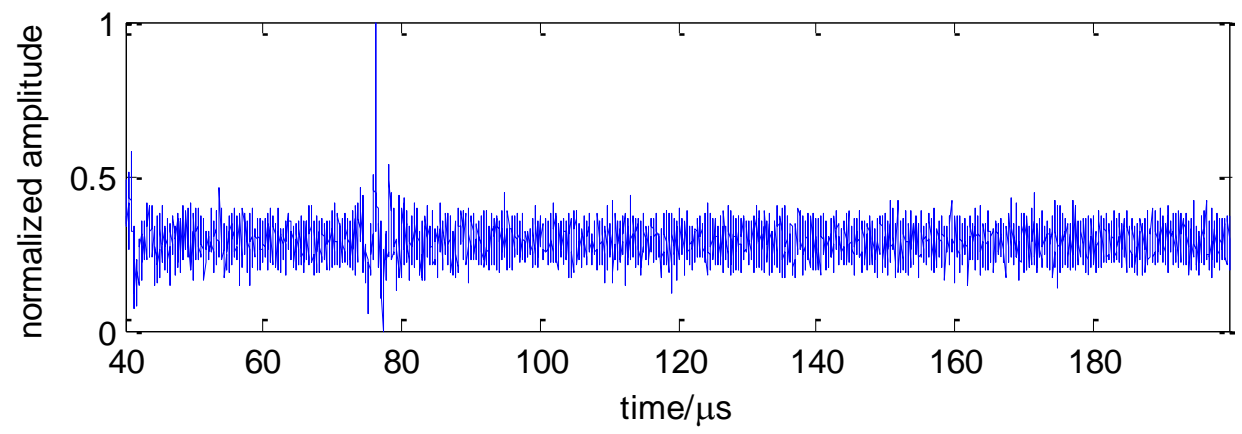

(b) Minimum Entropy Deconvolution

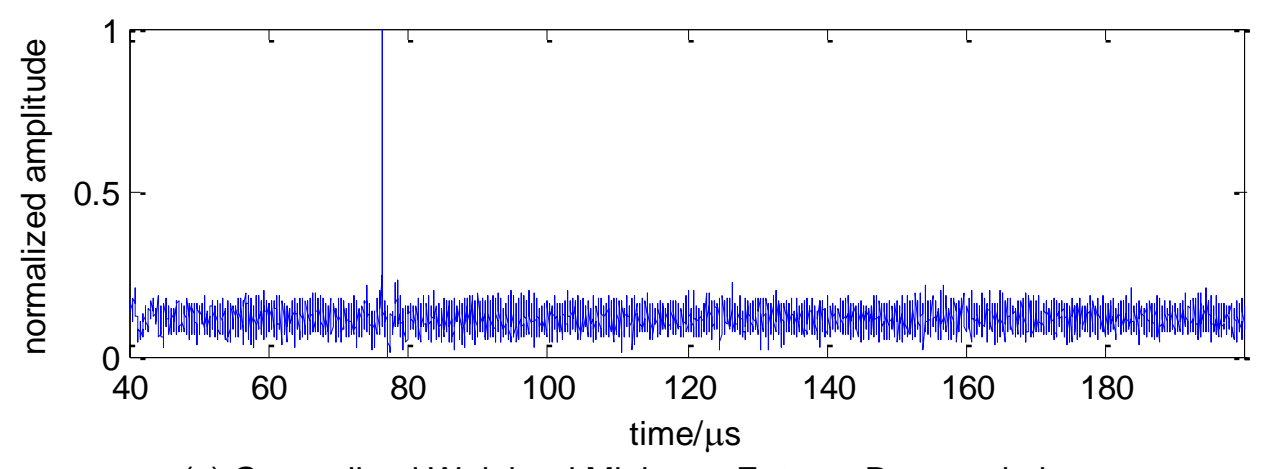

(c) Generalized Weighted Minimum Entropy Deconvolution

Figure 2. Original Signal and Several Deconvolution Results

Figures 2 (a), (b), (c), show that the resolution of the GWMED algorithm was improved significantly; the reflection coefficient estimation precision of the algorithm is higher than that of the MED algorithm. Based on the ultrasonic reflection sequence, the arrival time of the ultrasonic echo can be calculated. Because the ultrasonic guided wave has the characteristic of dispersion, the arrival time of the echo is the corresponding arrival time of the echo-peak. Figure 2, shows that the arrival time of the echo-peak is $75.14 \mu \mathrm{s}$. The ultrasonic echo arrival time of the MED algorithm is $76.18 \mu \mathrm{s}$, while that of the GWMED algorithm is $76.24 \mu \mathrm{s}$. The propagation speed of mode $\mathrm{L}(0,2)$ in the X70 pipeline steel is $3326 \mathrm{~m} / \mathrm{s}$. The axial position of defect is $124.958 \mathrm{~mm}, 126.688 \mathrm{~mm}$, and $126.787 \mathrm{~mm}$. Compared with the actual axial position of defect, the error of calculation are $3.042 \mathrm{~mm}, 1.312 \mathrm{~mm}$, and $1.213 \mathrm{~mm}$. Therefore, using the GWMED algorithm to calculate arrival time of echo is effective. Table 1, presents the iteration number of the different algorithms. Based on Table 1, the iteration number of the GWMED algorithm is significantly reduced compared with that of the MED algorithm. By adjusting the value of $a, b$, the iteration number can be reduced effectively. However, when $a, b$ is adjusted to a certain value, the iteration number tends to be saturated. 
Table 1. Iteration Number of the Different Deconvolution Algorithm

\begin{tabular}{|c|c|c|c|}
\hline & \multirow{2}{*}{$\begin{array}{l}\text { Minimum entropy } \\
\text { deconvolution } \\
\text { MED }\end{array}$} & \multicolumn{2}{|c|}{$\begin{array}{l}\text { Generalized weighted minimum entropy } \\
\text { deconvolution GWMED }\end{array}$} \\
\hline & & $a=100, b=1$ & $a=100, b=3$ \\
\hline iteration number & 13 & 6 & 4 \\
\hline
\end{tabular}

The kurtosis coefficients reflect the impact component of the signal. Thus, the kurtosis values are calculated with the results of certain deconvolution algorithm Figure 3. Figure 3 , shows that the kurtosis growth of the GWMED algorithm changes rapidly, which illustrates that a strong or weak component contrast variation of the reflective sequence is rapid. When $a=100, b=3$, and involving only 4 iterations, the same kurtosis value is obtained through the GWMED algorithm. Figure 2, shows the kurtosis value of all kinds of algorithm corresponding to each iteration.

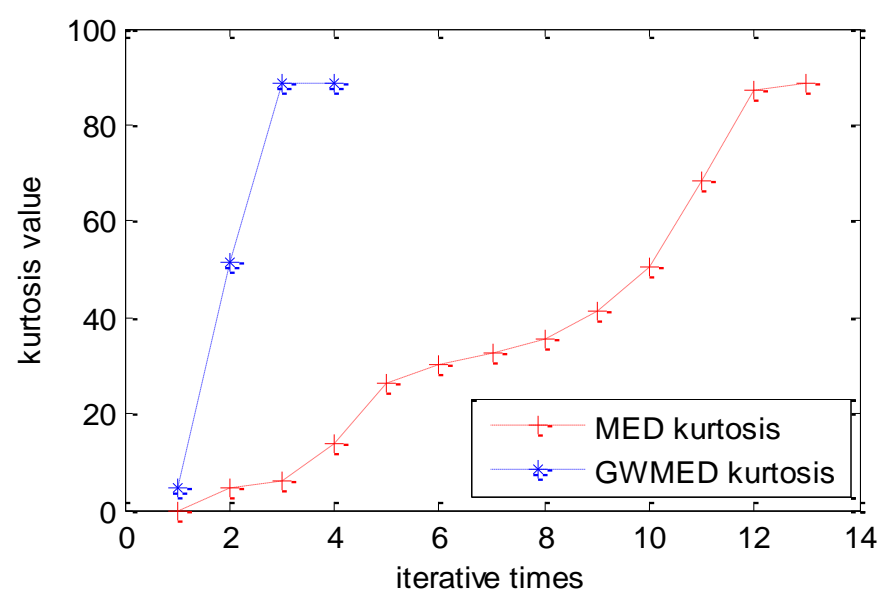

Figure 3. Relationship between Iteration Numbers and Kurtosis Value

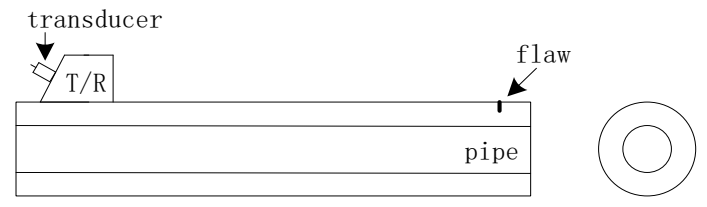

Figure 4. Schematic Diagram of Experiment Two

Figure 4, shows the schematic diagram of Experiment Two. The gas pipeline is 500 $\mathrm{mm}$ long, has a $273 \mathrm{~mm}$ diameter, and $8 \mathrm{~mm}$ wall thickness, and is made of X70 steel materials. The transducer and test equipment of Experiment Two is the same as those of Experiment One. Both experiments use the sampling frequency of $250 \mathrm{MHz}$. The defect is a prefabricated artificial crack, which is $25 \mathrm{~mm}$ in length, $3 \mathrm{~mm}$ wide, and $4 \mathrm{~mm}$ deep. Furthermore, the defect is $30 \mathrm{~mm}$ from the pipe end. Figure 5 (a), shows the measurement signal. 


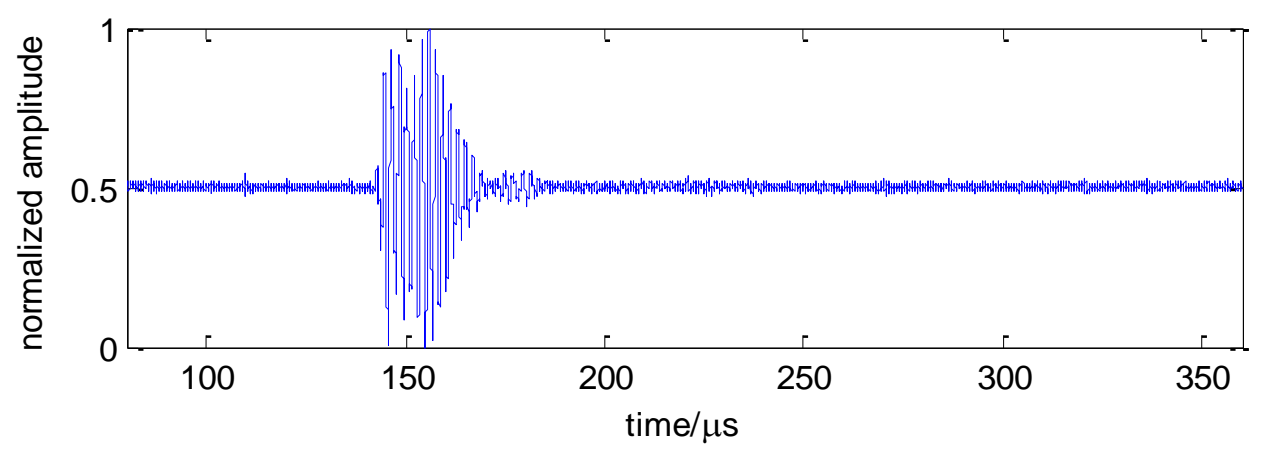

(a) Reflection Signal of the Defect and End Face

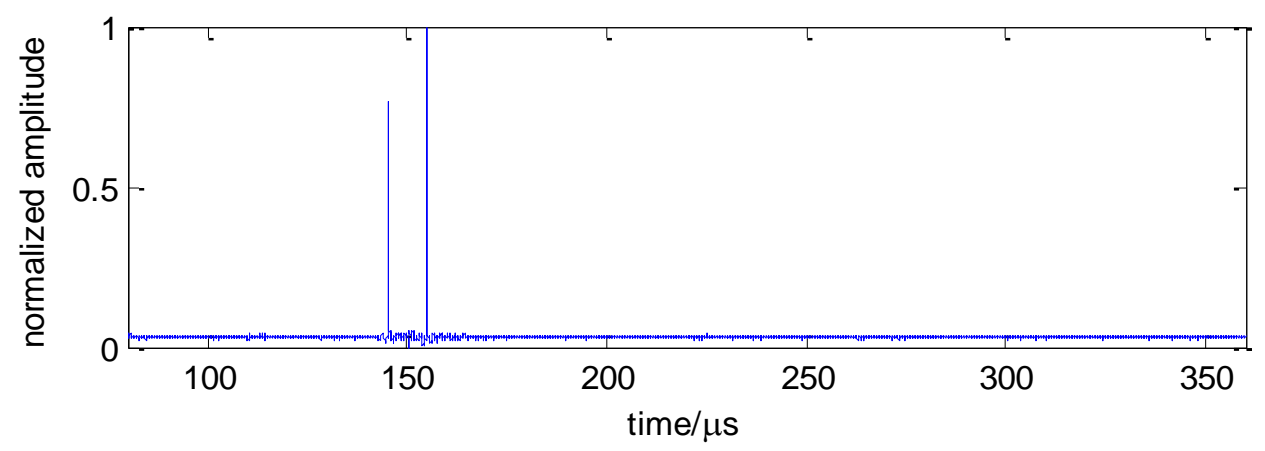

(b) Result of the Generalized Weighted Minimum Entropy Deconvolution

\section{Figure 5. Original Reflection Signal and Deconvolution Results of the Defect and End Face}

Figure 5, shows that the reflection signals of the defect and end face are overlapping, and cannot be distinguished. Using GWMED can obtain two clear sparse spikes. This result indicates that the algorithm can effectively distinguish between echo signals that are mixed together. Figure 5 (b), shows that the time difference of the two sharp pulses is 9.5 $\mu s$. The distance calculated by the wave velocity in the pipe is $31.597 \mathrm{~mm}$. The experiment proves that the GWMED algorithm can effectively improve the resolution.

\section{Conclusion}

This study presents an adjustable parameter of nonlinear weighted transform and combines with the MED algorithm, which can obtain the optimal deconvolution filter effectively. The algorithm has the better sparse results than MED. This study discusses the effect of the regulation parameter $a, b$ and kurtosis value.

The results indicate that making weighted nonlinear transform for sparse reflection sequence can effectively increase the contrast between the large sharp pulse and the small sharp pulse. Signal sparsity is stronger after the transformation, which suppresses the noise component on the deconvolution and improves the resolution of the signal. Moreover, the number of iteration is reduced effectively. The algorithm has a good effect on accurately obtaining TOA or TOF of the ultrasonic guide wave signal, as well as the accurate pinpointing of the defect.

\section{Acknowledgments}

This research work is supported by the National Natural Science Fund of China (ID number: 50975028). 


\section{References}

[1] M. J. S. Lowe, D. N. Alleyne and P. Cawley, "Defect detection in pipes using guided waves," Ultrasonics, vol. 36, no. 1-5, (1998), pp. 147-154.

[2] J. L. Rose, J. Mu and J. K. V. Velsor, "New directions in guided wave pipe testing," Materials Evaluation, vol. 65 , no. 4, (2007), pp. 375-378.

[3] J. L. Rose, "GUIDED WAVES Successes and Challenges for Ultrasonic Testing in NDT and SHM," Materials Evaluation, vol. 68, no. 5, (2010), pp. 495-500.

[4] T. Vogt, M. Lowe and P. Cawley, "The scattering of guided waves in partly embedded cylindrical structures," Journal of the Acoustical Society of America, vol. 113, no. 3, (2003), pp. 1258-1272.

[5] D. N. Alleyne, B. Pavlakovic, M. J. S. Lowe and P. Cawley, "Rapid, long range inspection of chemical plant pipework using guided waves", Advances in Nondestructive Evaluation, Pt 1-3, Key Engineering Materials S. S. Lee, D. J. Yoon, J. H. Lee and S. Lee, eds., Zurich-Uetikon: Trans Tech Publications Ltd, (2004), pp. 434-441.

[6] X. J. Wang, P. W. Tse and A. Dordjevich, "Evaluation of pipeline defect's characteristic axial length via model-based parameter estimation in ultrasonic guided wave-based inspection," Measurement Science \& Technology, vol. 22, no. 2, (2011), pp. 13.

[7] G. M. Zhang, D. M. Harvey and D. R. Braden, "Effect of sparse basis selection on ultrasonic signal representation," Ultrasonics, vol. 45, no. 1-4, (2006), pp. 82-91.

[8] W. Liang and P. W. Que, "Maximum non-Gaussianity parameters estimation of ultrasonic echoes and its application in ultrasonic non-destructive evaluation," Measurement Science \& Technology, vol. 18, no. 12, (2007), pp. 3743-3750.

[9] A. M. Sabatini, "Correlation receivers using laguerre filter banks for modelling narrowband ultrasonic echoes and estimating their time-of-flights," Ieee Transactions on Ultrasonics Ferroelectrics and Frequency Control, vol. 44, no. 6, (1997), pp. 1253-1263.

[10] R. Demirli and J. Saniie, "Model-based estimation of ultrasonic echoes part I: Analysis and algorithms," Ieee Transactions on Ultrasonics Ferroelectrics and Frequency Control, vol. 48, no. 3, (2001), pp. 787-802.

[11] W. Liang, H. M. Lei and P. W. Que, "Sparsity enhancement for blind deconvolution of ultrasonic signals in nondestructive testing application," Review of Scientific Instruments, vol. 79, no. 1, (2008), pp. 6.

[12] Y. H. Kim, S. J. Song and J. Y. Kim, "A new technique for the identification of ultrasonic flaw signals using deconvolution," Ultrasonics, vol. 41, no. 10, (2004), pp. 799-804.

[13] F. Lingvall, "A method of improving overall resolution in ultrasonic array imaging using spatio-temporal deconvolution," Ultrasonics, vol. 42, no. 1-9, (2004), pp. 961-968.

[14] R. A. Wiggins, "Minimum entropy deconvolution," Geoexploration, vol. 16, no. 1-2, (1978), pp. 21-35.

[15] X. Li, X. Li, W. Liang and L. Chen, "regularized minimum entropy deconvolution for ultrasonic NDT \&amp; E," NDT \& E International, vol. 47, no. 0, (2012), pp. 80-87.

[16] C. Jutten and J. Herault, "Blind Separation of Sources, An Adaptive Algorithm Based on Neuromimetic Architecture," Signal Processing, vol. 24, no. 1, (1991), pp. 1-10.

[17] T. Cicero, P. Cawley, F. Simonetti and S. I. Rokhlin, "Potential and Limitations of a Deconvolution Approach for Guided Wave Structural Health Monitoring," Structural Health Monitoring-an International Journal, vol. 8, no. 5, (2009), pp. 381-395.

\section{Authors}

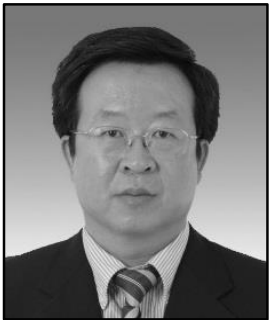

Haichao Cai, he received the BS and MS degrees in Mechanical Engineering from Henan University of Science and Technology, China, in 2003 and 2007, respectively. Now he is a $\mathrm{PhD}$ graduate in College of Mechanical Engineering, Beijing institute of Technology, China. Currently he is interested in Nondestructive detection and signal processing.

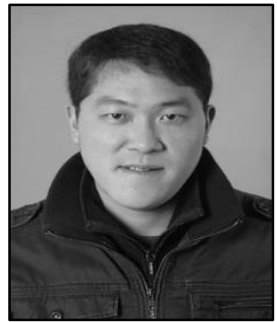

Chunguang Xu, Professor of Beijing Institute of Technology, director of Lab for Nondestructive Testing\&Control of Beijing Institute of Technolog, Standing director of Chinese Society of Nondestructive Testing, President of IEEE Far East NDT Conference, Director of Joint Lab for Ultrasonic NDT of Beijing Institute of Technology-America PAC.Prof. Xu has been engaged in the theory, technology, teaching and experimental studies of the 
acoustic emission, ultrasonics, structure laser measurement technology and automatic control. He had achieved over 130 papera and 60 innovative patents up to now.

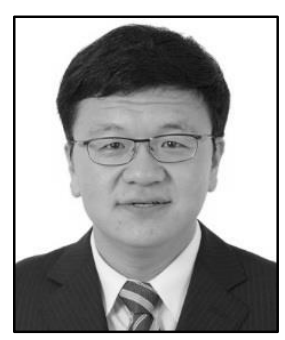

Qinxue Pan, He received the Ph.D in Intelligent Mechanical Engineering and System from Kagawa University, Japan. Currently, work at the school of Mechanical Engineering at Beijing Institute of Technology since 2010. The research interests in Mechanical Engineering, NDT\&E technology, Residual Stress Measurement and Characteristic Evaluation Technology of Stress Profile. As director or chief researcher carried out more than 5 projects, including National Natural Science Foundation of China, Ph. D. Programs Foundation of Ministry of Education of China.

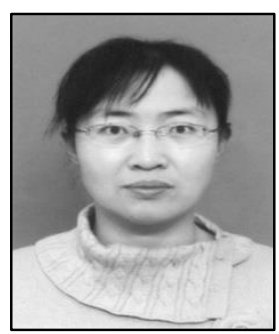

Hongjuan Yan, She received the $\mathrm{PhD}$ degrees in Mechanical Engineering from Beijing Institute of Technology, China, in 2015. Currently she is interested in ultrasonic nondestructive testing and signal processing. 